\title{
Abstract author index
}

World Federation of Critical Care Nurses, World Congress, Florence, Italy, 2009.

Web site: http://en.wfccn.org

Key words: abstracts * authors critcal care * index * nursing WFCCN *

\begin{tabular}{|c|c|c|c|}
\hline A & C & Demir F., 151 & Gori S., 162 \\
\hline & & Demirkilic U., 159 & Graham L., 154, 154 \\
\hline Abali S., 138, 140, 182 & Cachon J.M., 142, 143 & Demirtas A., 147, 147, 147, 150 & Green A., 153 \\
\hline Adams N., 166 & Cadman N., 149 & Diplou S., 158 & Griffiths J.A., 175 \\
\hline Aganon F., 138 & Cakmakci H., 151 & Dizer B., 182 & Grillo Padilha K., 155, 155 \\
\hline Aggelopoulou Z., 139,176, 176 & Cañón Montañez W., 143 & Donaldson P., 158 & \\
\hline $\begin{array}{l}\text { Aitken L., } 139 \\
\text { Akbayrak N. 147, } 147\end{array}$ & $\begin{array}{l}\text { Carmona Monge F.J., 139, 157, } \\
171\end{array}$ & Dragosev G., 163 & $\mathrm{H}$ \\
\hline Alameda Varela R., 147, 150, 171 & Carroll K., 143 & Dumbovic V., 150, 153 & Hadjibalassi M., 154 \\
\hline Albarran J., 153, 167 & Carter J., 182 & Duncan A., 172 & Haikali S., 167 \\
\hline Algora Weber A., 157 & Casey E., 144 & Durmaz Akyol A., 172 & Hayward A., 155 \\
\hline Alijevic S., 163 & Celotti P., 144 & $F$ & Herkes R., 171 \\
\hline Alvarez C., 142, 143 & Cerrillo Gonzalez I., 139, 157 & $E$ & Hirakawa N., 156 \\
\hline Antoniou A., 158 & 171 & Eager D., 150 & Houze P., 141 \\
\hline Argent A.C., 179 & Chaboyer W., 152, 164 & Eliott S., 151 & Hui Tzu Y., 156 \\
\hline Athini E., 154 & Chamberlain J., 149 & Elliott R., 150 & Hwang S.Y., 174 \\
\hline Aydemir H., 138 & Chang S., 144 & Endacott R., 169 & \\
\hline Aytacoglu I., 152 & Chauca P., 145 & Erasmus J., 155 & I \\
\hline & Chen S., 145 & Erasmus W.J., 155 & ledema R., 171 \\
\hline B & Chen Y., 146 & Erickson S., 172 & Ikematsu Y., 156 \\
\hline & Cheng W., 179 & Ernest D., 151 & Ito S., 157 \\
\hline Baktoft B., 153 & Chi S., 146 & Eti Aslan F., 151, 152 & Itojima Y., 157 \\
\hline Balci S., 138, 140, 182 & Chu-Ling C., 146 & & \\
\hline Bambi S., 175 & $\begin{array}{l}\text { Cicek H., 147, 147, 147, 150, } \\
181,182\end{array}$ & $\mathbf{F}$ & J \\
\hline Bana Derakhshan H., 140 & Cividini T., 148 & Fielding M., 152 & Jara Perez A., 139, 157, 171 \\
\hline Barlavie Y., 162 & Clark K., 158 & Finn J., 172 & Jennings J., 158 \\
\hline Bastos F., 140 & Claro C., 155 & Fletcher A., 182 & Jeong M.H., 174, 174 \\
\hline Baud F., 141 & Clayton S., 139 & Foster M., 152, 164 & Joutsalainen T., 158 \\
\hline Beca J., 168 & Coetzee M., 148 & Franco C.B., 168 & Juvan B., 142 \\
\hline Becattini G., 175 & Colbert K., 155 & Friganovic A., 153 & \\
\hline Bencivinni A., 165 & Collins T., 148 & Fulbrook P., 153 & K \\
\hline Bennett J., 154 & Conde A.R,, 138 & G & Kalafati M., 167, 167 \\
\hline Bento S., 155 & Corsini L., 162 & & Kallio N., 158 \\
\hline Bingham R., 180 & Coyer F., 149 & Gambirasi L., 154 & Karabinis A., 167 \\
\hline Birch C., 141 & Cremasco M., 169 & Garcia Gomez S., 139,157, 171 & Karadag Arli S., 151, 152 \\
\hline Blunt M., 182 & Crepaldi I., 184 & Geelhoed G., 172 & Karanikola M., 167, 167 \\
\hline Bonaconsa C., 148 & Curwood B., 149 & Georgiou E., 154 & Kashiwabara K., 176 \\
\hline Boykis Y., 175 & & Giachetta A., 1165 & Kent B., 168 \\
\hline Bozas E., 167 & D & Giannakopoulou M., 167, 167 & Kim J.H., 160 \\
\hline Brack S., 142, 164 & & Gillespie M., 174 & Kotila J., 177 \\
\hline Bracko V., 142 & $\begin{array}{l}\text { David A., } 141 \\
\text { Davies K. } 149\end{array}$ & Godfrey V., 165 & Kouimtzoglou A., 158 \\
\hline Brearley L., 180 & $\begin{array}{l}\text { Davies K., } 149 \\
\text { Davis A.H., } 146\end{array}$ & Goldsworthy S., 154, 154 & Koyuncu A., 159, 180, 181, 182 \\
\hline Burch V., 179 & De Monte A., 144 & Goncalves M.B., 163 & Koyuncu F., 159, 181 \\
\hline Burmeister E., 139 & & Gonzales R., 145 & Krieber H., 166 \\
\hline
\end{tabular}




\begin{tabular}{|c|c|c|c|}
\hline Krokoscz D.C., 155 & Monterosso L., 149 & \multicolumn{2}{|l|}{ Purisa S., 152} \\
\hline Kukolj G., 163 & Mori K., 180 & \multirow[b]{2}{*}{$\mathbf{Q}$} & \\
\hline Kurkluoglu M., 159 & Moritz R., 164 & & T \\
\hline Kwon H.K., 159 & Mourek K., 155 & \multirow{2}{*}{$\begin{array}{l}\text { Quiros Herranz C., 139, 157, } \\
\quad 171\end{array}$} & \\
\hline Kydonaki C., 159 & $\begin{array}{l}\text { Moylan T., } 164 \\
\text { Mpoti S., } 167\end{array}$ & & $\begin{array}{l}\text { Taguchi T., } 176 \\
\text { Takamizawa E., } 180\end{array}$ \\
\hline $\mathbf{L}$ & Mpouzika M., 167 & $\mathbf{R}$ & Takaoka H., 157 \\
\hline Ladanyi S., 150 & Msimeki N., 179 & \multirow{3}{*}{$\begin{array}{l}\text { Rajbhandari D., 149, } 171 \\
\text { Ramelet A., } 172\end{array}$} & Tallot M., 149 \\
\hline Lambrinou E 154 & Murphy N., 152 & & Taneike R., 176 \\
\hline Landeiro M. 160 & & & Temiz K., 152 \\
\hline Landeiro M.J., 160 & $\mathbf{N}$ & Reed P., 168 & Thomas J., 149 \\
\hline Langdon K., 172 & & Reid D., 182 & Tocina A.G., 163 \\
\hline Lappalainen A., 177 & Nafra A., 178 & Retallick L., 172 & Toffolleto M.C., 155 \\
\hline $\begin{array}{l}\text { Lee E.H., } 160 \\
\text { Lee E.S., } 174,174\end{array}$ & $\begin{array}{l}\text { Nagode Z., } 142 \\
\text { Nakamori M., } 176\end{array}$ & $\begin{array}{l}\text { Ribakovsky I., } 175 \\
\text { Rollan G., } 139\end{array}$ & $\begin{array}{l}\text { Tosun N., 147, 147, 147, 150, } \\
181,182\end{array}$ \\
\hline Lee S.H., 174 & Niccoli S., 165 & Rollan Rodriguez G., 157, 171 & Tsai J., 177,177 \\
\hline Lee S.K., 160 & Nicolaou E., 154 & Rowan P., 166 & Turkmen E., 182 \\
\hline Lehtinen J., 158 & Nigel Y., 175 & Rubin Y., 183 & \\
\hline Lemonidou C., 167 & Nishioka S., 176 & Ruiz de Luna R., 157 & $\mathbf{U}$ \\
\hline Leon V., 161 & Nocentini C., 162 & \multirow[t]{2}{*}{ Rush J., 154} & HIman Y 162 \\
\hline Leslie G.D, 149, 180 & Nusdorfer L., 165, 166 & & Ulman Y., 162 \\
\hline Levy G., 141 & \multirow[b]{2}{*}{$\mathbf{O}$} & $\mathbf{S}$ & Unlu D., 172 \\
\hline Liapi E., 161 & & Sabag M., 162 & V \\
\hline Libanori L., 184 & O'Brien S., 142 & Saban E., 172 & \\
\hline Lucey M., 171 & O'Connor S., 149 & Sachdeva S., 178 & Valovirta E., 177 \\
\hline Lyons A., 161 & O'Sullivan J., 149 & Sakata N., 157 & Vati J., 178, 178 \\
\hline M & Oggioni R., 165 & Sales A.S., 183 & Vivian L.M., 179, 179 \\
\hline IVI & Ong L.L., 160 & Samancioglu S., 172 & W \\
\hline Machado F., 164 & Ono P.M., 183 & Sareban M.T., 173 & \\
\hline Macleod L., 166 & Ozcan C.T., 147, 147, 150 & Saroglou G., 176 & Wakayama T., 176 \\
\hline Margazas A., 158 & \multirow{2}{*}{$\mathbf{P}$} & Satosek D., 142 & Watterson J., 141, 164 \\
\hline Marine K., 161 & & Sengul T., 173 & Wei L., 179 \\
\hline Marinelli N., 184 & Pacquola M., 166 & Serr N.F., 183 & Wenzel F., 169 \\
\hline Marsden K., 149 & Page K., 169 & Sewlall N., 155 & Whitaker I., 169 \\
\hline Martinez Lareo M., 139, 157, 171 & Palacios D., 142, 143 & Shackell E., 174 & Whitfield A., 151 \\
\hline Massey L., 172 & Pampel T., 166 & Sharma M., 178 & Williams T., 180 \\
\hline Matteucci L., 162 & Pang N.L., 160 & Sharma R., 178 & \multirow[b]{2}{*}{$\mathbf{Y}$} \\
\hline Mayo A., 162 & Papastavrou E., 154 & Shin E., 174,174 & \\
\hline McGuinness S., 168 & \multirow{2}{*}{$\begin{array}{l}\text { Papathanassoglou E., 167, 167, } \\
\quad 167,176\end{array}$} & Shnaider N., 175 & Yamada A., 180 \\
\hline McKay A., 179 & & Sidebottom B., 153 & \multirow{2}{*}{$\begin{array}{l}\text { Yava A., 147, 147, 147, 150, 159, } \\
\quad 180,181,182\end{array}$} \\
\hline McKinley S., 150 & Park S.A., 160 & Silva A., 140 & \\
\hline Mehrtens J., 149 & Parke R., 168 & Simmonds B., 171 & Yavuz M., 152 \\
\hline Menachem I., 162 & Patel R., 168 & Sipahi U., 173 & Yazici Korkmaz O., 138, 140, 182 \\
\hline Mendes S.H., 183 & Pedreira M.L., 168, 169 & Siviero E., 175 & Yeo M.W., 174 \\
\hline Menezes H., 166 & Peltokangas T., 158 & Smith J., 162 & Yeo M.Y., 171, 174 \\
\hline Merkouris A., 154 & Peressoni L., 144 & Solaro M., 175 & Yilmaz A., 173 \\
\hline Merrick E., 171 & Peris A., 175 & Sommacampagna M., 184 & Young P., 182 \\
\hline Migliaccio L., 175 & Perry J., 169 & Song M., 144 & \\
\hline Mills K., 162 & Peterlini M.A., 168 & Song M.S., 159 & $\mathbf{Z}$ \\
\hline Min-Ling L., 163 & Petrucci O., 163 & Spirou A., 176, 176 & Zambello B., 184 \\
\hline Miranda A.F., 163 & Pires A.M., 169 & Sproul S., 154 & Zambello D., 184 \\
\hline Mirkovic P., 163 & Pires R., 169 & Stafseth S., 176 & Zampier E., 183, 183 \\
\hline Mistarz R., 151 & Pirret A., 162, 170 & Stamler A., 183 & Zanei S., 169 \\
\hline Mitchell M., 164 & Plowright C., 148, 170, 170 & Stange C.E., 183 & Zanetti A., 144 \\
\hline Mitidieri A., 184 & $\begin{array}{l}\text { Poulish K., } 171 \\
\text { Prosperi E., } 162\end{array}$ & Swanepoel C.R., 179 & Zohar Z., 220 \\
\hline
\end{tabular}

